\title{
QuikScan Formatting as a Means to Improve Text Recall
}

\author{
Hans van der Meij ${ }^{1}$, Jan van der Meij ${ }^{1}, \&$ David Farkas ${ }^{2}$ \\ 1. \\ University of Twente \\ Faculty of Behavioural Sciences \\ Department of Instructional Technology \\ P.O. Box 217 \\ 7500 AE Enschede \\ The Netherlands
}

2.

University of Washington

Department of Human Centered Design \& Engineering

Campus Box 352315

Seattle, Washington (USA) 98195

*Requests for reprints should be addressed to Hans van der Meij, PO Box 217, 7500

AE Enschede, the Netherlands (e-mail: H.vanderMeij@utwente.nl). 


\begin{abstract}
Purpose - QuikScan is an innovative text format that employs three prominent signaling devices - summaries, headings, and access cues - to make the reading of medium-to-long texts more productive. The experiments reported in this paper examined the claim that QuikScan contributes to text recall.

Design - In two consecutive experiments a QuikScanned text (experimental condition) was compared to a non-QuickScanned text (control condition). In Experiment 1, 41 university students read the text and then answered ten open recall questions. In Experiment 2, 58 university students read the text and then wrote a summary and answered four recall questions.
\end{abstract}

Findings - In Experiment 1, a statistically significant overall effect on text recall favoring QuikScan was found. Detailed analyses revealed that QuikScan mainly affected the readers' responses to higher-order questions $(d=1.24)$. Experiment 2 showed that QuikScan led to significantly higher recall scores for the summaries. Just as in the first experiment, a strong effect on the higher-order questions was found ( $d$ $=1.27)$.

Research implications - Further studies of QuikScan should include studies in naturalistic settings and should address selective reading and information navigation as well as text recall. SARA, a recent comprehensive theory of signaling, makes it possible to identify the individual functions of QuikScan's signaling devices and conduct revealing studies of QuikScan.

Practical implications - QuikScan and other innovations that improve the reading experience can potentially increase the willingness of readers to read longer documents. 
QUIKSCAN FORMATTING AS A MEANS TO IMPROVE TEXT RECALL

Originality/value - QuikScan provides a unique combination of signaling devices. It can facilitate access and enhance text comprehension.

\section{Introduction}

At least as far back as the seventeenth century, readers have complained about the super-abundance of text (Robert Burton, Barnaby Rich, quoted in Carr, 2010). Francis Bacon (1625) went further and articulated a reading strategy to address this problem: While some books should be read thoroughly, others should be read lightly or only read in parts. More productive reading can also come about through better design, and indeed the history of literacy has been marked by a long succession of design innovations of text (Kilgour, 1998).

What about the present and what about the future? There is an increasing concern that many readers - especially knowledge workers - experience information overload (Eppler \& Mengis, 2004; Wurman, 1989) and don't have time to read everything they think necessary to keep up with their job duties and areas of expertise (Menzies \& Newson, 2007). For these individuals, browsing texts - a combination of scanning, skimming, and pausing for nuggets of information - has become a professional survival skill (Liu, 2005; Nicholas, Huntington, Jamali, Rowlands, Dobrowolski, \& Tenopir, 2008).

A related phenomenon is that online reading and the hypertextual nature of the web may reduce the willingness of readers to engage in the sustained reading of extended texts (Connolly, 2010; Nielsen, 2008). Moreover, persuasive arguments have been made that erosion of attention is reducing our collective ability to engage in deep reading (Carr, 2010; Connolly, 2010). 
QUIKSCAN FORMATTING AS A MEANS TO IMPROVE TEXT RECALL

One response to these changes in literacy is to create and publish a greater number of shorter texts. Indeed, publishers have been initiating publishing programs restricted to brief books (Amazon Singles, Springer Briefs, and Byliner.com). But assuming that many complex topics and issues continue to demand detailed textual exposition and that such texts must often be fully read and understood, we can perhaps look to innovative text design to counteract the resistance to sustained reading and, in so doing, to reduce the motivation on the part of publishers to ask authors to write shorter documents. Ideally we can encourage the use of longer documents by helping readers retain more for each hour of reading time they invest and by better supporting selective reading within a text.

\section{Innovations in text design}

One highly successful innovation, introduced in 1987, is the structured abstract (see Zhang \& Liu, 2011). When the first-level headings of a journal article or similar document are included within the article's abstract, readers retain more than when they read a document that begins with a conventional abstract. In addition, the headings enable readers to readily identify the section in the full document where an idea summarized in the abstract can be found. Another innovation, Visual-Syntactic Text Formatting, improves retention by breaking lines of text at phrase boundaries rather than at the right margin of the page (Walker, Schloss, Vogel, Gordon, Fletcher, \& Walker, 2007).

QuikScan, shown in Figure 1, is still another recent innovation that aims to make the reading of medium-to-long texts more productive (Zhou, 2008; Zhou \& Farkas, 2010). Sample QuikScan documents, published studies on QS, and related resources are available at www.QuikScan.org. 


\section{Diagnosis and resolution structure}

12\} Troubleshooting Procedures lead users through (brief or lengthy) phases of diagnosis and resolution activity.

13\} Important distinction: One or more methods comprising a distinct approach to solving a problem can be regarded as a solution path.

14\} The diagnosis phase may contain steps for resolution, and the resolution content may include diagnosis steps.

\{12 Almost all troubleshooting procedures lead the user through phases of diagnosis activity and resolution activity. Either phase can be brief (sometimes very brief) or lengthy. The diagnosis phase may consist of a single symptom or a complex configuration of symptoms. The resolution phase may consist of a single method (a set of steps to take) or many methods in a complex configuration. \{13 One important distinction is that one or more methods comprising a distinct approach to solving a problem can be regarded as a solution path.

Although the main structural pattern is diagnosis followed by resolution, $\{14$ at times the diagnosis phase may contain steps for resolution and the resolution content may include diagnosis steps.

Figure 1. A QuikScan summary (gray box) and an excerpt of the body text.

When a document is QuikScanned, multiple summaries are placed throughout the document. Readers, therefore, can choose between a summary and the full section of the document. These summaries are formatted as individual list items preceded by numbers. A key goal of the summaries is to improve retention. Furthermore,

QuikScan enables fine-grained navigation within the document. Readers interested in a summarized idea can easily scan for a corresponding "target" number placed in the text where the summarized idea is discussed in full (Zhou \& Farkas, 2009). Target numbers are visually salient because they are preceded by a left brace and because the number and brace are both boldfaced and highlighted in gray. In short, QuikScan potentially provides both a better way to read intensively and a better way for hurried or impatient readers to engage selectively with texts.

Zhou (2008) conducted an experiment in which 20 undergraduate students received a QuikScanned text, and 18 students read the same text without any summaries. The QuikScanned text consisted of 5,174 words; the control consisted of 4,393 words. The text discussed the topic of global trade, which was outside the scope 
QUIKSCAN FORMATTING AS A MEANS TO IMPROVE TEXT RECALL

of the students'area of study. Immediate text recall was assessed with a 17 -item multiple-choice test consisting of questions about concepts (e.g., "Which best defines ...?") and synthesizing questions that required higher-order information processsing (e.g., "Given ... what is the primary cause of ...?”). The results showed that the QuikScan group achieved a statistically significant higher overall score on the immediate text recall test than the control group. Cohen's (1988) $d$-statistic revealed that the difference between groups was 0.71 , which is considered a medium effect size. Differences for the two test question types were not reported.

Van der Meij and van der Meij (2011) conducted an experiment in which 20 undergraduate psychology students received a QuikScanned text, and 20 students received the same text preceded by a single, structured abstract but without the QuikScan summaries. The text was presented in English, the second language for the Dutch and German participants in the study. It was taken from a psychology textbook and described the phenomenon of flash bulb memory (FBM). The QuikScanned text contained 6,475 words; the control text contained 5,844 words. Immediate text recall was assessed with 12 open-ended questions that were divided into questions about facts (e.g., "What example is mentioned ... ?"), concepts (e.g., "What are the two most important differences between ...?"), and higher-order processes such as synthesis (e.g., "Why is the validity problematic when comparing ...?"). The results of the study showed that the students in the QuikScan condition had a statistically significant higher overall score on the recall test and the effect size was large $d=$ 1.57. The positive effect of QuikScan on text recall was also found to be present in all question types (i.e., $d_{\text {Fact }}=0.88 ; d_{\text {Concepts }}=0.70 ; d_{\text {Higher- } \text { Order }}=0.95$ ). 
QUIKSCAN FORMATTING AS A MEANS TO IMPROVE TEXT RECALL

\section{How does QuikScan yield better text recall?}

QuikScan was invented to solve the practical problem of how texts can better accommodate the processing of medium-to-long documents. To enhance text recall, QuikScan incorporates the qualities of three signaling devices that have been found to improve recall: overviews, headings, and enumeration/access cues. Signaling devices emphasize aspects of a text's organization or content without affecting that organization or content (Meyer, 1975) .

Empirical research shows that readers benefit from several related functions that overviews ${ }^{1}$ serve. First, an overview distinguishes text topics and provides concise labels for these topics. Second, an overview previews key information that is later elaborated in the text - the reader encounters the information twice. Third, an overview marks topics the author deemed important, and emphasizes their organization in a text (e.g., Hartley \& Trueman, 1985; Kintsch \& Van Dijk, 1978; Lorch \& Lorch, 1996a; Ritchey, Schuster, \& Allen, 2008; Sanchez, Lorch, \& Lorch, 2001; Spyridakis, 1989a, 1989b). The QuikScan summaries also serve these functions, but instead of doing so only once at the start of the text as is customary, QuikScan does this multiple times. QuikScan thus provides the reader with more information about the text structure, previews more items that are later elaborated in the text, and signals more topics as being important. In comparison with a single overview this should further enhance text recall.

Text recall may also be enhanced by the way in which QuikScan summaries are formatted and phrased. In contrast to the conventional summaries, QuikScan summaries generally appear directly below one of the document's headings and are formatted as separate numbered list items. Each list item is intended to express "one idea" and is phrased as a single sentence or, when necessary, two short, closely 
QUIKSCAN FORMATTING AS A MEANS TO IMPROVE TEXT RECALL

related sentences. Isakson and Spyridakis (1999) note that independent clauses (which are equivalent to sentences) are more effective as signals than dependent clauses.

These list items somewhat resemble document subheadings and - like subheadings - preview ideas at a lower level than a preceding heading. QuikScan summaries should therefore be expected to assist the reader in the construction of a schema and support subsequent text recall in very much the same way as headings do (e.g., Hartley, 1987; Hartley, Kenely, \& Trueman, 1980; Hyönä \& Lorch, 2004; Lorch \& Lorch, 1996a; Sanchez, et al., 2001; Spyridakis, 1989a, 1989b). In addition, whereas subheadings are scattered throughout the text, the list items in a QuikScan summary are presented together as an assembled set. This may have the additional advantage that they can be processed at a glance.

QuikScan summaries contribute to text recall in yet another way: by enumeration and access cueing. The list item numbers in the QuikScan summaries cue the reader about the sequence of key ideas. These numbers are structural markers that explicitly do what place position does implicitly, namely signal to the reader that an item high up in the summary list appears earlier in the text. Because this numbering scheme links summary statements to the topic structure manifested in the text, the target numbers, functioning as paratextual cues, may help certain categories of readers process text structure (e.g., Hyönä, Lorch, \& Kaakinen, 2002; Lorch \& Chen, 1986; Lorch, Lemarié, \& Grant, 2011a; Lorch \& Lorch, 1995, 1996b; Lorch, Lorch, Ritchey, McGovern, \& Coleman, 2001). This article describes two new studies that provide broader empirical support for QuikScan's effectiveness.Recently an important theoretical advance has been achieved in the area of text signaling: the SARA framework (Lemarié, Lorch, Eyrolle, \& Virbel, 2008). SARA, as we will argue in the general discussion, can lead to a more nuanced, theory-based studies of QuikScan. 


\section{Experiment 1}

This study was conducted with a different audience and study text than the studies by Zhou (2008) and van der Meij and van der Meij (2011). In the present study all the participants were Indonesian students, and all experimental materials as well as the participants' responses were written in their native language, Bahasa. Just as in Zhou's (2008) study, the text came from a domain outside of the participants' field of study.

The most important difference between the two earlier studies and this study is that the QuikScanned text has a more complex heading structure, an introductory overview, and three large graphics. In relation to the number of words in the text, there are substantially more headings in the Bahasa text than in the two previous studies. Because the Bahasa text has more headings and an introductory overview, it exhibits a more complex signaling environment. Because it has more headings, an overview, and graphics, it a more realistic text, more similar to typical texts that convey technical and professional information. A realistic study text is important because QuikScan is intended for use in professional settings.

Experiment 1 consisted of two conditions. A control group read the nonQuikScanned text. An experimental group read the QuikScanned text. In both conditions the original abstract from the text was included. There are two research questions:

Question 1: Is there a difference between conditions on measures of text perception? It is an open question whether the QuikScan text yields more positive appraisals of text quality measures (i.e., comprehensibility, structure, and interest) than the non-QuikScan text. In Zhou's (2008) study the participants expressed 
QUIKSCAN FORMATTING AS A MEANS TO IMPROVE TEXT RECALL

favorable opinions about QuikScan, but only the experimental group was questioned. Van der Meij \& van der Meij (2011), who directly compared the non-QuikScan and QuikScan conditions, found no differences between participants in their appraisals of text quality.

Question 2: Is there a difference between conditions on measures of text recall? The prediction is tested that for the more realistic and complex document the QuikScan condition yields a higher score on text recall.

\section{Method}

Participants. A total of 41 students (mean age 21.3 years) volunteered to participate in the study. All participants were studying in the Faculty of Agriculture at the Tanjungpura University, West Kalimantan, Indonesia. They were in their second, third, or fourth year of academic study. The students were randomly assigned to conditions with stratification for gender. In the control group there were 11 female and 10 male participants. In the experimental group gender was evenly distributed.

Instruments. The source text is a technical article on troubleshooting procedures for software users. It discusses how information is designed to support the user in problem diagnosis and resolution. The text is conceptual rather than empirical. It presents the findings from a linguistic analysis of troubleshooting procedures.

After the Abstract, the text is divided into five main sections by Level 1 headings (i.e., Introduction, Defining troubleshooting procedures, Developing troubleshooting procedures, Diagnosis \& resolution structure, and Conclusion). The section "Diagnosis \& resolution structure" is subdivided into two sections with Level 2 headings (i.e., The diagnosis phase, The resolution phase). The author, David 
QUIKSCAN FORMATTING AS A MEANS TO IMPROVE TEXT RECALL

Farkas, is one of the "inventors" of QuikScan. Consequently, the text included wellwritten QuikScan summaries.

The text used in the study presents the same content as the source, except for the removal of references to the literature. Also, the two-column formatting was changed to a one-column presentation. In the Control text all the QuikScan summaries and the target numbers in the text were removed.

The Control text consists of 4,117 words that, together with the three original Figures, was presented on 11 pages. The QuikScan text included 10 summaries for a total of 40 summarizing statements. Together these elements added another 879 words, yielding a QuikScan text of 14 pages.

Pilot testing indicated that a maximum of 45 minutes of reading time would suffice for most students. A questionnnaire gathered information about participant characteristics and background, such as gender, age, and year of study. Checks on randomization revealed an even distribution for age and year of study.

Another questionnnaire assessed the participants' appraisal of text comprehensibility (e.g., "I had no problem understanding this text" and "The text was comprehensible"), text structure (e.g., "I liked the way the text was formatted" and “The text was well structured") and topical interest (e.g., "I found the topic appealing" and "I have become curious about the topic of the text"). The questionnaire consisted of 18 statements. The translated version was pilot tested with 30 students who did not take part in the study. To answer these questions, the participants could tick off a point on a Likert scale which ranged from completely disagree (1) to completely agree (7).

The recall test consisted of 10 open-ended questions. Seven of these called for conceptual knowledge (e.g., "Define troubleshooting" and "What is the purpose of the 


\section{QUIKSCAN FORMATTING AS A MEANS TO IMPROVE TEXT RECALL}

diagnosis phase?"). The other three questions called for higher-order information processing such as analysis and synthesis. These questions required some (re)ordering of information, or combining several concepts into a still broader idea (e.g.,"What is the function of modularization in the resolution phase?" and "Mention three ways in which troubleshooting procedures differ from standard procedures"). The questions concerned important text topics from the beginning, middle section and end of the text. The questions were traceable to one or more QuikScan summary statements, but students needed to have read the main body text to construct a complete answer.

Coding was done with the aid of a codebook which presented the main information units that each answer should contain and the scoring for these units. The maximum score for each information unit was set at 0.20 points. In other words, the points for a correct answer to a question varied, depending on the number of information units in the answer. For instance, the answer to the question "What analogy is used to describe how troubleshooting works?" consists of one unit, namely "medical treatment". Therefore, the maximum score for this question was 0.20 points. Similarly, a complete answer to "Please define troubleshooting" would contain five information units and therefore yield a maximum of $5 * 0.20$ points. For the whole test students could receive a maximum of 7.80 points. For the two subtypes of questions in the test, this broke down into a maximum of 5.20 for the seven conceptual questions and a maximum of 2.60 points for the three higher-order questions.

Two native speakers pretested the codebook by independently scoring a set of recall tests obtained from a pilot study. The results were discussed and the codebook was adjusted where needed. This procedure was repeated until the coders agreed. After interobserver agreement was established and consolidated in the codebook, the 
QUIKSCAN FORMATTING AS A MEANS TO IMPROVE TEXT RECALL

summaries were scored by three coders who were not aware of the participants' condition while scoring. The Recall tests of the study were scored by the same coders.

Procedure. Participants were told that the study examined the influence of text design on comprehension and recall. Participants were asked to carefully study the text for a maximum of 45 minutes, or less if they felt they were done. Note taking was not allowed. Thereafter, they would be asked to answer recall questions. After reading, participants first filled in the two questionnaires and then took the recall test.

Data analysis. Analyses of the questionnaire data from the participants indicated that reliability increased after the removal of one question for each of the quality measures. The resulting outcome was satisfactory reliability for comprehensibility, interest, and structure with Cronbach alpha scores of respectively 0.75, 0.77, and 0.73. An ANOVA showed that reading time was virtually identical in both conditions, $F<1$, n.s. Students with the Control text needed an average of 43 minutes of reading time, while students with the QuikScan text spent an average of 42 minutes reading. A large majority of participants, 16 in the control and 13 in the experimental condition, spent the full 45 minutes reading the text.

A MANOVA preceded the analyses of variance on the comparisons for the three text-perception quality measures and the two item types in the recall test. All analyses were two-sided with alpha set at 0.05 . For effect size we report Cohen's (1988) $d$-statistic. These scores tend to be defined as small $(d=0.20)$, medium $(d=$ $0.50)$, and large $(d=0.80)$.

\section{Results}

In regard to perceptions of text quality, the MANOVA indicated that there was no statistically significant difference between conditions for the three measures of text 
QUIKSCAN FORMATTING AS A MEANS TO IMPROVE TEXT RECALL

quality, $F(3,36)=1.17$, n.s. In short, both texts received essentially the same rating from the participants. The absolute levels (see Table 1) all tend to be quite close to the neutral mean score of 3.5. This indicates that the participants found neither text extremely good or bad on comprehensibility or structure, and neither text to be of high or low interest.

Table 1. Means (Standard Deviation) for measures of text perception ${ }^{1}$.

\begin{tabular}{lcc}
\hline & Control Condition & QuikScan Condition \\
\hline Comprehensibility & $2.79(1.00)$ & $3.28(1.26)$ \\
Structure & $3.71(0.90)$ & $4.17(1.24)$ \\
Interest & $3.81(1.15)$ & $4.51(1.18)$
\end{tabular}
${ }^{1}$ Scores on text quality measures were given on a 7-point scale; higher scores reflect higher
appreciation.

QuikScan does not yield an important advantage for judgements on text quality. This finding also signals that it is unlikely that differences in text recall can be ascribed to differences in perceptions of text quality.

Scores on the text recall questions are shown in Table 2. An ANOVA indicated that the difference between the two conditions on the overall text recall score was statistically significant, $F(1,40)=4.21, p=0.047, d=0.32$. The effect size statistic indicates that the difference is small.

Table 2. Means (Standard Deviation) for text recall questions.

\begin{tabular}{ccc}
\hline & Control Condition & QuikScan Condition \\
\hline Overall recall $(\max 7.8)$ & $2.04(0.78)$ & $2.57(0.88)$ \\
Conceptual $(\max 5.2)$ & $1.68(0.72)$ & $1.85(0.73)$ \\
Higher-order $(\max 2.6)$ & $0.36(0.21)$ & $0.72(0.35)$ \\
\hline
\end{tabular}


QUIKSCAN FORMATTING AS A MEANS TO IMPROVE TEXT RECALL

A MANOVA also indicated that there was a statistically significant difference between conditions for the two types of recall questions, $F(2,38)=7.80, p=0.001$. Subsequent ANOVAs showed that the scores for conceptual questions were comparable across conditions, $F<1$. In contrast, there was a statistically significant difference for higher-order questions. Students in the QuikScan condition gave significantly more correct answers to these questions than students in the control condition, $F(1,40)=16.0, p<0.001, d=1.24$. The effect size statistic signals the presence of a large difference.

\section{Discussion}

Experiment 1 replicates the earlier finding that QuikScan significantly affected text recall. Where van der Meij and van der Meij (2011) reported a positive effect of QuikScan on all question types, the present study found this only for higher-order questions. The scores for these two item types in the recall test indicate that the effect is relatively minor for conceptual questions whose answers were easily available in the text. The main effect of QuikScan on text recall pertains to the students' capacity to answer higher-order questions. Answering these questions hinges on processes such as analysis and synthesis, on making connections between different text elements or sections.

\section{Experiment 2}

To further examine the claim that QuikScan enhances text recall, a second experiment was conducted. Experiment 2 employed the same two conditions: a non-QuikScan control group and a QuikScan group. Moreover, the same text was used with a similar audience. 
QUIKSCAN FORMATTING AS A MEANS TO IMPROVE TEXT RECALL

The main difference between the two experiments concerns the text recall measure. Experiment 1 assessed text recall through open-ended questions. In Experiment 2 participants were asked to write a summary. More so than answers to an experimenter's questions, such summaries measure topic structure representations. They measure how well participants remember the main themes in the text (e.g., Brooks, Dansereau, Spurlin, \& Holley, 1983; Hyönä \& Lorch, 2004; Lorch, Lorch, \& Inman, 1993; Lorch, et al., 2001; Sanchez, et al., 2001). The participants were also asked to answer four open-ended questions from the recall test of Experiment 1. This measure was used to roughly compare the outcomes of the two studies, and to examine the relationship between the two measures of recall. Perceptions of text quality were no longer measured. The central research question in Experiment 2 is: Is there a difference between conditions on measures of text recall?

\section{Method}

Participants. A total of 58 students (mean age 19.5 years) volunteered to participate in the study. All participants were students in their second year of study for an accounting major in the Faculty of Economics and Social Sciences at the State Islamic University of Sultan Syarif Kasim, Riau, Indonesia. Students were randomly assigned to conditions with stratification for gender. In the control condition there were 20 female and 9 male participants. In the experimental condition there were 19 female and 10 male participants. Just as in Experiment 1, all materials and reponses were in the student's native language (i.e., Bahasa).

Instruments. The texts for the Control and QuikScan conditions were the same as in Experiment 1. Because many students in Experiment 1 finished reading close to the 
QUIKSCAN FORMATTING AS A MEANS TO IMPROVE TEXT RECALL

maximum allotted time, reading time was extended by 5 minutes. All students chose to keep on reading for the full 50 minutes of the study.

A questionnnaire gathered information about participant characteristics and background, such as gender, age, and year of study. Checks on randomization revealed an even distribution for age and year of study.

Participants were given a blank sheet of paper on which to write their summary. A pilot test revealed that participants could complete writing their summary within 20 minutes. The written summaries were coded with the aid of a codebook. The information units in the codebook were derived from the summaries of the participants and then validated against the text. In the summaries all information was divided into items (information units). Each item presented in the summary that was also in the text would yield a 1.0 score. For instance, the original text contains a list of four items on the nature of troubleshooting procedures. This means that the maximum score of this list in the summary would be 4 points. The total score of a participant's summary is thus simply the sum of all points, with more points reflecting more items in the summary. After establishing reliability (i.e., Cronbach alpha 0.90), the summaries were scored by three coders who were not aware of the participants' condition while scoring.

We computed four scores for the summaries: items, QuikScan items, nonQuikScan items, and Abstract items. The item measure is simply a sum of all the items presented in the participants' summaries. The measure indicates how many ideas were given. The QuikScan item measure indicates the degree to which items that were present in the QuikScan summaries were included in the participants' summaries. Because the QuikScan summaries contain the most significant (superordinate) ideas in the text, a high-quality summary will contain more of these 
QUIKSCAN FORMATTING AS A MEANS TO IMPROVE TEXT RECALL

ideas and fewer of the lower-level non-QuikScan items (i.e., all items not found in the QuikScan summaries). The Abstract item measure indicates the degree to which items that were present in the Abstract of the text were included in the participants' summaries. The Abstract items represent the most important ideas from the text. Just like the QuikScan items measure, this measure also provides an excellent yardstick for gauging the quality of the participants' summary.

A recall test contained 4 of the 10 text recall questions from Experiment 1. Two questions called for conceptual knowledge (max. 1.4), and two for higher-order information processing $(\max 2.0)$. As in Experiment 1, an overall test score was computed, as well as a score for item type. Scoring was the same as in Experiment 1.

Procedure. Participants were told that they were participating in a study on reading in which "we are interesting in finding out what people remember from studying a text when they are not allowed to make notes or mark the text in any way". In contrast to Experiment 1 participants were not told that the study examined text design. They were asked to carefully study the text for a maximum of 50 minutes after which they would be asked to write a summary and answer a few text questions. After reading the text, the participants filled in the Background questionnaire and were given 20 minutes to write their summary. Finally, the four recall questions were answered. Data analysis. A MANOVA preceded the analyses of variance on the comparisons for the two item types in the recall test. All analyses were two-sided with alpha set at 0.05 .

\section{Results}

The scores for the quality of the participants' summaries in each of the two conditions are shown shown in Table 3. An ANOVA indicated that the QuikScan condition 
QUIKSCAN FORMATTING AS A MEANS TO IMPROVE TEXT RECALL

yielded a higher score for the number of items than the Control condition, $F(1,57)=$ $7.25, p=0.009, d=0.71$. The effect size indicates a medium effect on this measure of the extension or topic coverage of the participants' summary. Table 3 further details the outcomes for the three item types: QuikScan items, non-QuikScan items, and Abstract items.

Table 3. Means (Standard Deviation) for items in participants' summaries.

\begin{tabular}{lcc}
\hline & Control Condition & QuikScan Condition \\
\hline Number of items & $6.28(3.58)$ & $9.14(4.46)$ \\
Number of QuikScan items & $4.48(2.29)$ & $8.59(4.18)$ \\
Number of non-QuikScan items & $1.79(2.48)$ & $0.55(0.98)$ \\
Number of Abstract items & $3.14(2.49)$ & $5.21(2.48)$ \\
\hline
\end{tabular}

In both conditions the presence of QuikScan items in the summaries is significantly and substantially higher than the presence of non-QuikScan items, $t_{\text {control }}$ $(28)=4.58, p<.000, d=1.23 ; t_{\mathrm{qs}}(28)=10.51, p<.000, d=2.65$. In other words, in both conditions the participants' summaries consisted primarily of ideas that can be found in the QuikScan summaries and fewer of the lower-level ideas that do not appear in these summaries.

An ANOVA further revealed that in the QuikScan condition the participants' summaries included significantly more of the items from the QuikScan summaries than in the control condition, $F(1,57)=21.49, p=0.000, d=1.22$. That is, summaries in the QuikScan condition were of higher quality. The effect size is large.

On the non-QuikScan items, the opposite was found. In the control condition the participants' summaries included more (low level) ideas not found in the QuikScan summaries, $F(1,57)=6.26, p=0.015, d=0.66$. However, it should be 
QUIKSCAN FORMATTING AS A MEANS TO IMPROVE TEXT RECALL

noted that in this comparison the assumption of homogeneity of variances was violated which cautions against drawing firm conclusions about this finding.

The bottom row in Table 3 shows the results for the Abstract items. The findings again favor the QuikScan group, which had a significantly higher score than the control group, $F(1,57)=10.04, p=0.002, d=0.83$. The effect size is large.

Scores for the text recall questions are shown Table 4. Just as in Experiment 1, an ANOVA showed that the difference between the two conditions on the overall text recall score was statistically significant, $F(1,57)=12.46, p=0.001, d=0.95$. The effect size is large.

Table 4. Means (Standard Deviation) for text recall questions.

\begin{tabular}{ccc}
\hline & Control Condition & QuikScan Condition \\
\hline Overall recall $(\max 3.4)$ & $0.44(0.18)$ & $0.68(0.31)$ \\
Conceptual $(\max 1.4)$ & $0.19(0.15)$ & $0.17(0.14)$ \\
Higher-order $(\max 2)$ & $0.26(0.14)$ & $0.51(0.25)$ \\
\hline
\end{tabular}

The MANOVA indicated that there was a statistically significant difference between conditions for the two types of recall questions, $F(2,55)=11.63, p<0.001$. Subsequent ANOVAs showed that the scores for conceptual questions were comparable across conditions, $F<1$. In contrast, there was a statistically significant difference for higher-order questions. Students in the QuikScan condition gave significantly more correct answers to these questions than students in the control condition, $F(1,57)=23.20, p<0.001, d=1.27$. The effect size statistic indicates that the difference is large. These findings are in line with the outcomes obtained in Experiment 1. 
QUIKSCAN FORMATTING AS A MEANS TO IMPROVE TEXT RECALL

\section{Discussion}

Experiment 2 replicated the finding from Experiment 1 for open-ended questions. In addition, there was support for the effectiveness of QuikScan on the participants' written summaries. Together, the two studies provide good evidence that QuikScan improves recall. Positive results have been obtained with different study populations, different study texts, and different measures for recall. In all instances, the difference between conditions was statistically significant and substantial with medium-to-large effect sizes.

\section{General Discussion}

This section consists of two parts. First we explain how a new and comprehensive theory of signaling called SARA (Lemarié et al., 2008) - Signaling that is $\underline{\text { Available, }}$

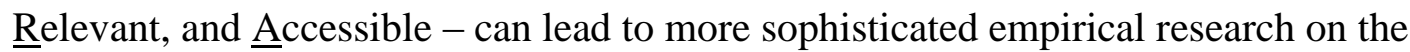
functionality of QuikScan's signaling devices. Then we propose extending the study of QuikScan by through naturalistic studies that test all the goals that QuikScan was designed to achieve.

\section{QuikScan and SARA signaling theory}

How does QuikScan influence recall? This is a difficult question because QuikScan consists of a combination of signaling devices (i.e., overviews, quasi-headings, and enumeration and access cues) that are also employed in multiple ways. Furthermore, QuikScan is intended to support multiple kinds of reading behavior (i.e., reading for recall, reading summaries instead of document sections, and quickly locating the full discussion of specific ideas found in the summaries). Indeed, because it employs a 
QUIKSCAN FORMATTING AS A MEANS TO IMPROVE TEXT RECALL

combination of signaling elements and serves multiple functions QuikScan is an elaborate form of signaling.

To better understand the multi-faceted nature of the signaling in QuikScan, SARA (Lorch, et al., 2011a; Lorch, Lemarié, \& Grant, 2011b) can be helpful. SARA proposes that a clear distinction be made between six main functions of signaling devices. That is, signals can demarcate, organize, label, typify, identify the topic, and emphasize by indicating importance.

Signals can demarcate. They can indicate the structural boundaries or junctures in a text. For instance, a heading and the indentation of a new line of text are commonly used to cue the reader of the beginning of a new text segment. QuikScan summaries do the same. They alert the reader to the presence of a new section of the text.

Signals can organize. They can inform the reader about the relationships between parts of a text. Overviews and headings generally communicate the hierarchical relationships of the text topics. Sequential relationships may be signaled with enumerations or lists. The numbered QuikScan summary statements - quasiheadings - may serve this function.

Signals can label. They can give the reader a concise name or description of another part of the text, serving more or less like an index entry term. Signals with this functionality include overviews, headings, and preview sentences. The individual QuikScan summary statements may also function in this way.

Signals can typify. They can communicate to the reader that a section is an introduction, provides background information, or defines a concept. A structured abstract (Hartley, 2004, 2008) with its divisions by information types (e.g., aim, background, method, results, conclusion) is a perfect example. QuikScan summaries 
QUIKSCAN FORMATTING AS A MEANS TO IMPROVE TEXT RECALL

can be optimized for this function, say, by adding a preview word before the summary statement itself (e.g., "Introduction. Troubleshooting procedures (TPs) are important and highly prevalent in the computer industry.").

Signals can identify the topic. They can inform the reader about a theme or concept. This function resembles that of typifying. The difference is that typifying is classificatory, while signals that identify the topic present the reader with specific, substantive information. The function can be served with headings, QuikScan summaries, and by using italics when a concept is introduced.

Signals can emphasize. All signals can draw the reader's attention to some degree. But by “emphasis" Lemarié et al. (2008) mean a more specific functionality, namely a signal that communicates that some part of the text is especially relevant or important. Emphasizing can be done in preview sentences, with key words, and by visual means such as by extra white space before and after a text fragment.

SARA's taxonomy enables the design of revealing studies that can establish whether each of the information functions of signaling is separable and has a demonstrably differential effect on the reader. That is, the researcher can choose the function in which he or she is interested and then devise a text and a study in which signals perform that specific function. For instance, a study on QuikScan's organizational functions could focus on the QuikScan numbering system. Recall that each statement (list item) in a QuikScan summary is preceded by a number, and that all the statements collectively yield an enumeration that cues the reader about the sequencing of items presented immediately below the summary. Experiments on this organizational function would test this element of QuikScan against alternatives such as bulleted lists or color coding (Lorch, et al., 2011a) with an appropriate user task such as asking readers to engage in a search task in which information about 
QUIKSCAN FORMATTING AS A MEANS TO IMPROVE TEXT RECALL

sequential organization would prove useful. The prediction then is that the experimental condition yields the best outcomes because it offers the best

organizational signal to the reader. SARA, then, can serve as the theoretical basis for a new series of QuikScan studies. At the same time, these studies would demonstrate how well SARA can illuminate highly complex forms of signaling.

\section{Extending the study of QuikScan}

The two Bahasa studies reported here plus earlier studies we have cited indicate that QuikScan has shown promise in improving text recall. However, QuikScan was designed to support multiple reading strategies: navigation and selective reading as well as text recall. Therefore, future studies of QuikScan should encompass more than text recall. Also, because readers, when engaged in actual work, shift reading strategies, we need to test QuikScan in naturalistic settings (workplace or school) to understand how readers utilize QuikScan while engaging in actual work. Naturalistic studies, unlike controlled laboratory experiments, are sensitive to the complex learning goals, motivations, and time pressures that arise in actual use but are hard to simulate in a controlled study.

One goal of QuikScan is to support fine-grained navigation within a document: to allow readers to jump easily from a summarized idea to the full discussion of that idea in the body of the document. However, this claim is supported by only one study (Zhou \& Farkas, 2009), and this is a focused, laboratory-type experiment in which readers were given search tasks.

Another key goal of QuikScan is to enable busy or impatient readers to save time by choosing to read summaries rather than sections of the document, but we do 
QUIKSCAN FORMATTING AS A MEANS TO IMPROVE TEXT RECALL

not know nearly enough about how readers would actually make these choices and what the consequences might be.

Naturalistic studies can be conducted through observation, retrospective thinkaloud protocols (where readers comment on videos of the tasks they have carried out), instrumented versions of QuikScan, and gaze tracking. These studies should include readers with lower levels of reading ability than university students or well-educated professionals engaged in knowledge work. Lower-ability readers might benefit greatly from QuikScan, assuming that they can make use of QuikScan's complex signalling environment.

\section{Conclusion}

Even if new studies confirm the claims that QuikScan benefits reading in regard to text recall, navigation, and support for selective reading, there remains the large gap between successful innovation and adoption: Many beneficial innovations never take hold. Structured abstracts align closely with the familiar practice of employing abstracts at the beginning of journal articles and structured abstracts require only a small amount of extra effort and a very small increase in the size of the document. In contrast, QuikScan requires a much larger effort in regard to text summarization and document formatting and significantly increases document length. However, while QuikScan requires more extensive modifications to documents than structured abstracts, it offers a wider range of benefits. In addition to improving comprehension and recall, QuikScan supports selective reading of a document at a fine level of granularity.

These benefits may not be sufficient to persuade the publishers of thousands of academic journals and similar documents to adopt QuikScan, but it might be 
QUIKSCAN FORMATTING AS A MEANS TO IMPROVE TEXT RECALL

sufficient to motivate a presentation of QuikScan documents in certain settings. For example, if a particular journal article was required reading in a medical school curriculum, the school might obtain the original word processing file (plus copyright permission) from the publisher of the journal, include QuikScan in the document, and make it available to students. A textbook publisher hoping to enter a lucrative but competitive niche in the education marketplace might do likewise (especially if it were an eTextbook) to provide a competitive advantage.

Corporations and other organizations might employ editors to QuikScan highvalue corporate documents. When QuikScan was initially envisioned, its main purpose was to improve the effectiveness of business meetings in which a document was the focus of discussion (Zhou \& Farkas, 2006). Because attendees often fail to read or carefully read documents in advance of meetings, QuikScan was intended to allow these attendees to participate meaningfully in the discussion because they could both read the summaries and scan to the relevant sections of the full documents. This use of QuikScan, while never empirically tested, remains compelling.

Finally, we should keep in mind that the key features of QuikScan might be carried into newer and better designs and that technology can potentially reduce the extra effort entailed in creating QuikScanned documents. Even now, very simple shortcut techniques in MS Word significantly ease the time spent in formatting the summaries. More could be done using MS Office's built-in programming language, Visual Basic for Applications. Furthermore, continuing advances in the computer science field of automated text summarization (a branch of computational linguistics) may greatly reduce the effort entailed in summarization. If societal changes are indeed reducing the time we have for reading and even our willingness to read extended texts and if we continue to assume that many societal issues demand detailed 
QUIKSCAN FORMATTING AS A MEANS TO IMPROVE TEXT RECALL

understanding, the benefits of QuikScan and other innovations that improve reading should be worth a significant amount of extra effort.

\section{Footnotes}

1. We use the terms abstract, overview and summary interchangeably. In this article, an overview generally stands for a text that is presented before the main body text. In referring to empirical studies we also concentrate on overviews that preview, rather than review, a text.

\section{Acknowledgements}

The authors wish to thank Farid Zeno Muhammad, Fauzan Ansyari Muhammad, Isnaëni Mukhammad, Vascolino Pattipeilohy, \& Suhendri for their help in conducting the experiment. We are very grateful for the helpful comments of two anonymous reviewers.

\section{References}

Bacon, F. (1625). Of Studies. Essays or counsels, civil and moral. In B. Vickers (Ed.), Francis Bacon: the major works (pp. 439-340). New York: Oxford University Press, USA, 2002.

Brooks, L. W., Dansereau, D. F., Spurlin, J. E., \& Holley, C. D. (1983). Effects of headings on text processing. Journal of Educational Psychology, 75, 292-302.

Carr, N. (2010). The shallows: What the Internet is doing to our brains, New York, NY: WW Norton \& Company.

Cohen, J. (1988). Statistical power analysis for the behavioral sciences, Hillsdale, NJ: Erlbaum Associates. 
QUIKSCAN FORMATTING AS A MEANS TO IMPROVE TEXT RECALL

Connolly, R. (2010). What's wrong with online reading? Retrieved from http://www.slideshare.net/randyconnolly

Eppler, M. J., \& Mengis, J. M. (2004). The concept of information overload: A review of literature from organization science, accounting, marketing, MIS, and related disciplines. The Information Society, 20, 325-344.

Hartley, J. (1987). Designing electronic text: The role of print-based research.

Educational Technology Research and Development, 35, 3-17. doi: 10.1007/BF02769448

Hartley, J. (2004). Current findings from research on structured abstracts. Journal of the Medical Library Association, 92, 368.

Hartley, J. (2008). Academic writing and publishing: a practical guide. Abingdon: Routledge.

Hartley, J., Kenely, G. O., \& Trueman, M. (1980). The effect of headings on children's recall from prose text. British Journal of Educational Psychology, $40,304-307$

Hartley, J., \& Trueman, M. (1985). A research strategy for text desigbers: The role of headings. Instructional Science, 14, 99-155.

Hyönä, J., \& Lorch, R. F. (2004). Effects of topic headings on text processing: Evidence from adult readers' eye fixation patterns. Learning and Instruction, $14,131-152$

Hyönä, J., Lorch, R. F., \& Kaakinen, J. K. (2002). Individual differences in reading to summarize expository text: Evidence from eye fixation patterns. Journal of Educational Psychology, 94, 44-55.

Isakson, C. S., \& Spyridakis, J. H. (1999). The influence of semantics and syntactics on what people remember. Technical Communication, 46, 366-381. 
QUIKSCAN FORMATTING AS A MEANS TO IMPROVE TEXT RECALL

Kilgour, F. G. (1998). The evolution of the book. New York: Oxford University Press. Kintsch, W., \& Van Dijk, T. A. (1978). Toward a model of text comprehension and production. Psychological Review, 85, 363-394.

Lemarié, J., Lorch, R. F., Eyrolle, H., \& Virbel, J. (2008). SARA: A text-based and reader-based theory of signaling. Educational Psychologist, 43, 27-48.

Liu, Z. (2005). Reading behavior in the digital environment: Changes in reading behavior over the past ten years. Journal of Documentation, 61, 700-712.

Lorch, R. F., \& Chen, A. H. (1986). Effects of number signals on reading and recall. Journal of Educational Psychology, 78, 263-270.

Lorch, R. F., Lemarié, J., \& Grant, R. A. (2011a). Signaling hierarchical and sequential organization in expository text. Scientific Studies of Reading, 15, 267-284.

Lorch, R. F., Lemarié, J., \& Grant, R. A. (2011b). Three information functions of headings: A test of the SARA theory of signaling. Discourse Processes, 48, 139-160.

Lorch, R. F., \& Lorch, E. P. (1995). Effects of organizational signals on textprocessing strategies. Journal of Educational Psychology, 87, 537-544.

Lorch, R. F., \& Lorch, E. P. (1996a). Effects of headings on text recall and summarization. Contemporary Educational Psychology, 21, 261-278.

Lorch, R. F., \& Lorch, E. P. (1996b). Effects of organizational signals on free recall of expository text. Journal of Educational Psychology, 88, 38-48.

Lorch, R. F., Lorch, E. P., \& Inman, W. E. (1993). Effects of signaling topic structure on text recall. Journal of Educational Psychology, 85, 281. 
QUIKSCAN FORMATTING AS A MEANS TO IMPROVE TEXT RECALL

Lorch, R. F., Lorch, E. P., Ritchey, K., McGovern, L., \& Coleman, D. (2001). Effects of headings on text summarization. Contemporary Educational Psychology, $26,171-191$.

Menzies, H., \& Newson, J. (2007). No time to think: Academics' life in the globally wired university. Time and Society, 16, 83-98.

Meyer, B. J. F. (1975). The organization of prose and its effects on memory. Amsterdam: North-Holland.

Nicholas, D., Huntington, P., Jamali, H. R., Rowlands, I., Dobrowolski, T., \& Tenopir, C. (2008). Viewing and reading behaviour in a virtual environment: The full-text download and what can be read into it. Aslib proceedings, 60, 185-198.

Nielsen, J. (2008). How little do users read? Retrieved from www.useit.com/alertbox/percent-text-read.html

Ritchey, K., Schuster, J., \& Allen, J. (2008). How the relationship between text and headings influences readers' memory. Contemporary Educational Psychology, $33,859-874$.

Sanchez, R. P., Lorch, E. P., \& Lorch, R. F. (2001). Effects of headings on text processing strategies. Contemporary Educational Psychology, 26, 418-428.

Spyridakis, J. H. (1989a). Signaling effects: A review of the research - part I. Journal of technical writing and communication, 19, 227-240.

Spyridakis, J. H. (1989b). Signaling effects: Increased content retention and new answers - Part II. Journal of technical writing and communication, 19, 395415. 
QUIKSCAN FORMATTING AS A MEANS TO IMPROVE TEXT RECALL

van der Meij, H., \& van der Meij, J. (2011). Improving text recall with multiple summaries. British Journal of Educational Psychology. doi: 10.1111/j.20448279.2011.02024.x

Walker, R. C., Schloss, P., Vogel, C. A., Gordon, A. S., Fletcher, C. R., \& Walker, S. (2007). Visual-Syntactic Text Formatting: Theoretical basis and empirical evidence for impact on human reading. IEEE IPCC proceedings, 1-14.

Wurman, R. S. (1989). Information Anxiety. New York: Doubleday.

Zhang, C., \& Liu, X. (2011). Review of James Hartley's research on structured abstracts. Journal of Information Science, 37, 570-576. doi:

$10.1177 / 0165551511420217$

Zhou, Q. (2008). QuikScan: Facilitating document use through innovative formatting. University of Washington, Seattle, USA.

Zhou, Q., \& Farkas, D. K. (2006). QuikScan: An innovative approach to support document use in meetings. Paper presented at the 2006 STC Annual Conference, Las Vegas, Nevada.

Zhou, Q., \& Farkas, D. K. (2009). QuikScan: Facilitating reading and information navigation through innovative document formatting. Paper presented at the IEEE International Professional Communication Conference, Hawaii, USA.

Zhou, Q., \& Farkas, D. K. (2010). QuikScan: Formatting documents for better comprehension and navigation. Technical Communication, 57, 197-209. 